\title{
Terapia cognitiva baseada em mindfulness no atendimento clínico individual de depressão*
}

\section{Nazaré de Oliveira Almeida ${ }^{1,2}$ \\ (D) https://orcid.org/0000-0001-8610-3753 \\ Marcelo Demarzo ${ }^{3}$ \\ (D) https://orcid.org/0000-0002-7447-1834 \\ Carmem Beatriz Neufeld ${ }^{1}$ \\ (ID) https://orcid.org/0000-0003-1097-2973}

Objetivo: estudo de casos múltiplos teve objetivo de avaliar a aplicabilidade do protocolo de grupo de um programa de 8 semanas de Terapia Cognitiva Baseada em Mindfulness adaptado para o atendimento clínico individual de pacientes com sintomas de depressão. Método: para o estudo de casos múltiplos, no qual 11 casos de pacientes com diagnóstico de depressão leve à moderada submetidos à intervenção adaptada desse protocolo foram avaliados nos resultados de medidas de depressão, ansiedade, estresse e mindfulness. Resultados: indicaram redução significativa destes sintomas, bem como aumento de mindfulness na vida cotidiana. Conclusão: os benefícios da intervenção de Terapia Cognitiva Baseada em Mindfulness podem se apresentar tanto no contexto de atendimento clínico grupal, bem como no individual. Neste estudo, Todos os participantes do estudo tiveram melhora significativa dos sintomas de depressão, bem como dos níveis de ansiedade e estresse, condizente com a os dados apresentados na literatura. É premente a necessidade de maior número de estudos desta natureza. Pesquisas futuras, especialmente as de natureza empírica, controlados e randomizados, consolidarão a validade externa dos resultados encontrados por estudos com a natureza como o deste.

Descritores: Atenção Plena; Meditação; Depressão; Ansiedade.

\section{Como citar este artigo}

Almeida NO, Demarzo M, Neufeld CB. Mindfulness-Based Cognitive Therapy in individual clinical care for depression. SMAD, Rev Eletrônica Saúde Mental Álcool Drog. 2020;16(3):55-63. doi: https://dx.doi.org/10.11606/issn.1806-6976.smad.2020.165509 


\section{Mindfulness-Based Cognitive Therapy in individual clinical care for depression}

Objective: the aim of this multiple case study was to to evaluate the applicability of the group protocol of an 8-week Mindfulness-Based Cognitive Therapy program adapted for the individual clinical care of patients with symptoms of depression. Method: the method used was to study multiple cases, in which 11 cases of patients with a diagnosis of mild to moderate depression who underwent intervention adapted from this protocol were evaluated in the results of measures of depression, anxiety, stress and mindfulness. Results: the results indicated a significant reduction in these symptoms, as well as an increase in mindfulness in everyday life. Conclusion: the benefits of the Mindfulness-Based Cognitive Therapy intervention can be presented both in the context of group clinical care, as well as in the individual. In this study, all study participants had significant improvement in the symptoms of depression, as well as in the levels of anxiety and stress, consistent with the data presented in the literature. There is an urgent need for a greater number of studies of this nature. Future research, especially those of an empirical nature, controlled and randomized, will consolidate the external validity of the results found by studies with nature such as this one.

Descriptors: Mindfulness; Meditation; Depression; Anxiety.

\section{Terapia cognitiva basada en la atención plena en la atención clínica individual para la depresión}

Objetivo: el objetivo de este estudio de caso múltiple fue evaluar la aplicabilidad del protocolo grupal de un programa de terapia cognitiva basada en la atención plena de 8 semanas adaptado para la atención clínica individual de pacientes con síntomas de depresión. Método: el método utilizado fue estudiar múltiples casos, en el que 11 casos de pacientes con un diagnóstico de depresión leve a moderada que se sometieron a una intervención adaptada de este protocolo fueron evaluados en los resultados de las medidas de depresión, ansiedad, estrés y atención plena. Resultados: los beneficios de la intervención de Terapia Cognitiva Basada en Mindfulness se pueden presentar tanto en el contexto de la atención clínica grupal, como en el individual. En este estudio, todos los participantes del estudio tuvieron una mejoría significativa en los síntomas de la depresión, así como en los niveles de ansiedad y estrés, consistente con los datos presentados en la literatura. Existe una necesidad urgente de un mayor número de estudios de esta naturaleza. Las investigaciones futuras, especialmente las de carácter empírico, controladas y aleatorizadas, consolidarán la validez externa de los resultados encontrados por estudios de naturaleza como este.

Descriptores: Atención Plena; Meditación; Depresión; Ansiedad. 


\section{Introdução}

A depressão consiste, na atualidade, em um dos maiores problemas de saúde pública e de risco por morte prematura(1), com um aumento de $18,4 \%$ no período entre 2005 e 2015 em todo o mundo, a elevando ao patamar de condição alarmante, com 4,4\% de prevalência no mundo. Em termos percentuais, o Brasil aparece com o maior índice da América Latina e o quinto maior do mundo, com prevalência de 5,8\%.

Um dos maiores desafios da depressão é sua recorrência em mais de um episódio. Diversas guidelines, por exemplo, American Psychiatric Association (APA), Canadian Network for Mood and Anxiety Treatments (CANMAT), Cochrane e The National Institute for Health and Care Excellence (NICE), apoiam-se em dados de pesquisas de intervenções específicas para o primeiro episódio e para depressão recorrente, sendo o tratamento, neste último caso, de manutenção. Uma destas intervenções atualmente muito utilizada e pesquisada é a Mindfulness-Based Cognitive Therapy (MBCT), desenvolvida em 2002 para depressão recorrente $^{(2)}$. Ela se baseia na integração da Terapia Cognitivo-Comportamental (TCC) para a depressão(3) com o Programa de Redução de Estresse Baseado em Mindfulness (MBSR) desenvolvido por Kabat-Zinn e colegas $^{(4)}$. Nesta integração, muitos elementos foram mantidos, mas outros alterados e acrescidos. Há na MBCT uma ênfase na mudança da consciência em relação ao eu e aos estados de pensamento, emoção, sensações e impulsos, mas nenhuma ênfase na alteração do conteúdo dos pensamentos(5).

Um pressuposto fundamental da MBCT é que o cultivo de um relacionamento consigo descentrado dos estados mentais autodepreciativos, funcionando como prevenção em momentos de potencial recaída. Neste sentido, o principal meio de desenvolver este descentramento é ensinando as pessoas a se tornarem mais conscientes dos estados mentais e se relacionarem com eles em uma perspectiva mais ampla, reconhecendo-os como eventos mentais apenas e não se identificando com eles ou reconhecendo-os como necessariamente reais ${ }^{(2-5)}$. A MBCT inclui uma variedade de práticas de mindfulness cujo objetivo é a observação e aceitação das sensações corporais, percepções, cognições e emoções sem julgamento ou reação. Estes estados são vistos como a natureza própria da mente.

O uso de Mindfulness está associado à redução de $44 \%$ do risco de recaída em pacientes com histórico de três ou mais recaídas de sintomas depressivos(6). A MBCT pode ser usada para reduzir sintomas residuais da depressão não importando a quantidade de episódios depressivos do paciente, apresentando efeitos comparáveis a permanecer em uma dose de manutenção de antidepressivos ${ }^{(7)}$. Para pessoas que buscam uma abordagem psicossocial para manterem-se saudáveis, Mindfulness aparece como uma alternativa acessível, aceitável e de baixo custo. Baseado nessas evidências, o guia sobre depressão do National Institute for Health and Clinical Excellence ${ }^{(8)}$ passou a recomendar Mindfulness para pessoas que estão atualmente bem, mas, já tiveram três ou mais episódios de depressão.

Estudos(2) $^{(2)}$ acerca dos resultados do uso de Mindfulness em pacientes com histórico de depressão apontam que a descoberta mais importante de sua pesquisa foi que, nos participantes com três ou mais episódios anteriores de depressão (que constituiu mais de $75 \%$ dos pacientes estudados), a MBCT diminuiu praticamente as taxas de recaída/recorrência em 50\% durante o período de acompanhamento comparado aos sujeitos que receberam um tratamento usual. Como os pacientes foram tratados em grupos, em termos de custo-efetividade, esse benefício é muito maior em comparação com os pacientes tratados individualmente, no formato terapia cognitiva individual convencional da depressão. Com efeito, a TCBM foi capaz de auxiliar até 12 pacientes praticamente no mesmo tempo que é necessário para tratar um único paciente em uma única sessão convencional de terapia cognitiva da depressão.

Programas MBCT foram submetidos à estudos de metanálises ${ }^{(9-11)}$ com tamanho de efeito comparável a intervenções reconhecidamente recomendadas devido ao nível de evidência de efetividade para esta população, por exemplo, a TCC(12). Elas apresentaram resultados significativos na prevenção de recaídas na depressão, bem como reduzindo sintomas depressivos em populações não clinicas $^{(13)}$.

Estudos do protocolo de grupo já utilizados no contexto individual(14) realizaram um primeiro ensaio clínico randomizado controlado com pacientes diabéticos (tipo 1 e 2) com sintomas depressivos $(\mathrm{N}=12)$, obtendo resultados de significativa efetividade da intervenção para estes sintomas, além de melhora em estados de ansiedade, bem-estar, mindfulness e autocompaixão. O estudo adaptou o clássico protocolo MBCT de 8 semanas $^{(2)}$ para um programa individual para pessoas com depressão e diabetes. Segundo os autores, nesta adaptação foi mantida a estrutura, conteúdos básicos e tarefas de casa do programa de grupo original. No próximo ano, o mesmo grupo de autores realizou outro estudo controlado e randomizado(15), comparando os efeitos de intervenções individuais de TCC e MBCT com uma lista de espera em uma ampla amostra de pacientes com o mesmo perfil $(\mathrm{N}=91)$. Os resultados mostraram e qualidade na efetividade de ambas intervenções na redução de sintomas depressivos. Em seguida, os autores ${ }^{(14-16)}$ realizaram novo estudo controlado e randomizado a partir do estudo anterior, com o objetivo de avaliar efeitos de longo prazo de uma amostra extraída 
da mesma amostra anterior, neste caso, de pacientes que mantiveram sintomas depressivos. Os resultados na redução de sintomas depressivos mostraram efetividade da intervenção em longo prazo (9 meses).

A maioria das pessoas com depressão preferem tratamento psicológico que medicamentoso(17). Além disso, algumas preferem intervenções individuais que grupais(18-19). Entretanto, a literatura acerca da MBCT para o contexto individual é muito escassa. Provavelmente que haja vantagens e desvantagens, tanto em uma quanto em outra abordagem. Parece que o compartilhamento de experiências e encorajamento constituem as principais vantagens nos grupos, ao passo que a privacidade e a personalização na abordagem individual. Além disso, pode ocorrer casos em que para o perfil de cada pessoa seja indicada uma ou outra alternativa, de modo que se ela se submete a uma menos indicada, pode sofrer menos ou até nenhum efeito benéfico esperado. Contudo, estas condições não foram amplamente verificadas, o que torna estudos como este pertinente.

O presente estudo visa estudar a aplicabilidade do protocolo de grupo de um programa de 8 semanas de Terapia Cognitiva Baseada em Mindfulness, adaptado do modelo original desenvolvido para grupos $^{(2)}$ para o atendimento clínico individual de pacientes com sintomas de depressão. A hipótese inicial é que tal intervenção possa promover redução de sintomas de depressão, ansiedade e estresse, bem como aumento de mindfulness na vida cotidiana. Além disso, espera-se que o protocolo de grupo possa ser adaptado para o contexto de atendimento clínico individual.

\section{Método}

Em relação ao delineamento de pesquisa, trata-se de um estudo de casos múltiplos. Diferente de um estudo de caso único, preocupa-se mais em estabelecer as similaridades entre situações, estabelecendo uma base para generalização, o que muitas vezes justifica a generalização de um caso para outro, muito mais do que para uma população de $\operatorname{casos}^{(20-21)}$. O estudo foi realizado em consultório particular de um dos autores, na cidade de Foz do Iguaçu.

A amostra deste estudo foi de conveniência e consistiu de 11 pacientes que procuraram atendimentos psicoterápicos da clínica do primeiro autor durante o ano de 2018 e atenderam os critérios de inclusão e exclusão do estudo. O fluxo de pacientes dessa clinica é em média 57 por ano.

Os critérios de inclusão para os participantes deste estudo foram: (a) idade maior que 18 anos; (b) disponibilidade de participar de pelo menos 10 sessões de terapia no horário e local especificados; (c) sintomas de depressão maiores do que o normal e isso exige, no mínimo, uma pontuação de 78 em percentil no instrumento DASS-21. Como critério de exclusão foi adotado: (a) sintomas severos ou extremamente severos de depressão, ou seja, uma pontuação maior que de percentil maior que 95 em percentil no instrumento DASS-21; (b) prática rotineira prévia de meditação.

Segundo especialistas em análise qualitativa(22), deve-se tomar um cuidado essencial no critério de escolha dos casos que é evitar um estreitamento do universo para escolha, quando não se analisam casos que estejam na fronteira do fenômeno que se pretende analisar. Para os autores, os casos de fronteira podem evidenciar aspectos que não foram inicialmente considerados, oferecendo assim dados para comparação de discrepâncias que forçam o pesquisador a clarear os conceitos e confirma os limites estabelecidos para escolha da amostra. Desta maneira, seguindo o critério de população beneficiada pelo programa, os pacientes estavam no mínimo no terceiro episódio de depressão e fora de crise.

A seleção foi realizada por meio de entrevista clínica baseada no DSM-5(23) e uso do instrumento DASS-21 e pelo próprio psicoterapeuta o qual conduziu a psicoterapia adaptada da intervenção do protocolo de 8 semanas de $\mathrm{MBCT}^{(2)}$. Os outros autores pesquisadores participaram das etapas que sucederam a essas.

Os participantes que concluíram a intervenção e avaliação deste estudo são 6 do sexo feminino e 5 do sexo masculino, com idades que variaram entre 19 anos e 57, todos brasileiros e com nível superior de escolaridade completo ou em curso, sem nenhuma experiência prévia com meditação. Um participante saiu do grupo por indisponibilidade de tempo, visto que mudou de horário de trabalho.

Tanto para a inclusão e exclusão de participantes neste estudo quanto para avaliação de melhora de sintomas de depressão, ansiedade e estresse foi utilizada a Depression Anxiety and Stress Scale - DASS21 , instrumento que consiste em um conjunto de três subescalas cada uma contendo 7 itens do tipo Likert, de 4 pontos, de autoresposta. Cada subescala é destinada a avaliar os estados emocionais de depressão, ansiedade e estresse. O respondente deve indicar o quanto cada item aplicou-se a ele durante a última semana. Os itens da escala likert referem-se à gravidade ou frequência e são organizados numa escala de 0 a 3 pontos sendo que o resultado é obtido pelo somatório das respostas aos itens que compõem cada uma das três subescalas. Foi adaptada para o contexto brasileiro em 2014(24), os quais verificaram uma boa consistência interna: os valores do alfa de Cronbach foram, respectivamente, de 0,90 para a depressão, 0,86 para a ansiedade, 0,88 para o estresse e 0,95 para o total das três sub-escalas. 
Também foi utilizado neste estudo a Mindful Attention Awareness Scale - MAAS, que é composta por 15 itens, com o objetivo de avaliar a atenção voltada para a consciência do momento presente e o aspecto atencional da atenção plena. Os autores conceituam mindfulness como um atributo da consciência relacionado ao bem-estar, composto por dois fatores, consciência e atenção, apesar de operacionalizarem o construto unifatorialmente: atenção mindfulness. Os respondentes devem indicar o quanto têm experimentado o que está descrito em cada uma das afirmativas, usando uma escala de seis pontos, que varia de um (quase sempre) a seis (quase nunca). Altos escores refletem maior capacidade de mindfulness. A escala foi desenvolvida por Brown e Ryan em 2003 e validada por Barros et al. ${ }^{(25)}$ que encontraram a consistência interna de alfa de Cronbach $=0,83$.

Quanto aos procedimentos, na primeira sessão propõe-se o protocolo para os pacientes e é preenchido os instrumentos de avaliação e TCLE, bem como é realizada a psicoeducação da $\mathrm{MBCT}$, com ênfase nos modos mentais fazer e ser. Nesta sessão é esclarecida a importância das práticas diárias em casa, sem as quais a eficácia da intervenção é comprometida. Durante 8 sessões no mínimo e, no caso dos 11 participantes, 18 sessões no máximo, foram realizadas as 8 sessões adaptadas do protocolo do manual do terapeuta Mindfulness-based cognitive therapy for depression: a new approach to preventing relapse, $2^{a}$ ed.(2) e no manual do paciente The Mindful Way Workbook An 8-Week Program to Free Yourself from Depression and Emotional Distress (ibid., 2014). A adaptação se referia ao número de práticas realizadas em sessão ou o tipo e quantidade de elementos da psicoeducação em cada sessão, dependendo da necessidade do paciente. Após a realização da última sessão adaptada do referido protocolo, procedeu-se a avaliação pós intervenção.

A intervenção consiste na adaptação do programa original de grupo para o contexto individual apresentou algumas distinções, as quais se referem principalmente às necessidades e características pessoais de cada paciente e ao tempo da sessão. As sessões duraram $1 \mathrm{~h}$, enquanto no programa de grupo duram $2 \mathrm{~h}$. Desta forma, a adaptação foi feita reduzindo a duração das práticas em sessão, mas mantendo o número de práticas e inquiry. Além disso, as características e necessidades pessoais levaram à uma variação na distribuição do tempo para cada atividade em sessão. Outro aspecto que diferenciou as sessões individuais deste estudo se referiu à psicoeducação que contemplou aspectos distintos do tema depressão, ansiedade e estresse, dependendo da presença de outros aspectos clínicos como comorbidades ou outros problemas de saúde, gravidade dos sintomas, características e necessidade pessoais, nível sócio-cultural, interesses pessoais e estilo de vida. Vale ressaltar que as práticas de casa se mantiveram fiéis ao proposto no programa original de grupos.

Quanto aos conteúdos básicos, estrutura das sessões e tarefas de casa, a adaptação manteve os mesmos propostos pelo programa original para grupos. Eles são apresentados a seguir, sessão a sessão.

Na primeira sessão do protocolo, cujo tema é "Além do Piloto Automático", é realizada em sessão a "prática da uva-passa", "escaneamento corporal" e em seguida o inquiry, técnica de abordagem do instrutor de MBCT, realizada imediatamente após as práticas, a qual visa estimular a conscientização do paciente acerca de suas experiências presentes. O protocolo propõe a prática de três exercícios durante a primeira semana: "escaneamento corporal", trazer consciência para as atividades rotineiras e "comer consciente".

$\mathrm{Na}$ segunda sessão do protocolo, de tema "Outra Maneira de Conhecimento", é realizada em sessão a "prática da respiração na posição sentada" e o inquiry. O protocolo propõe como atividades da semana o "escaneamento corporal", "breve exercício de respiração", trazer consciência para as atividades rotineiras e o calendário de experiências agradáveis. Entre outros assuntos, aborda-se o poder dos pensamentos e sentimentos, a noção de que pensamentos não são fatos - são eventos mentais e como o humor afeta a interpretação dos fatos e essa interpretação afeta o humor. O protocolo sugere enquanto de prática de casa: "escaneamento corporal", trazer consciência para as atividades rotineiras e agenda das experiências agradáveis.

Na terceira sessão do protocolo, com temática "Voltando ao Presente - Reunindo a Mente Dispersa", são propostas as práticas de "alongamento" e "espaço de respiração de 3 minutos", seguidas do inquiry. 0 protocolo sugere enquanto de prática de casa quatro atividades para a semana: prática combinada de alongamento e respiração intercalada com a prática do movimento consciente, espaço de respiração de 3 minutos e agenda das experiências desagradáveis, com o objetivo de ajudar o indivíduo a acalmar e reorganizar a mente inquieta e dispersa.

Na quarta sessão do protocolo, cuja temática é "Reconhecendo a Aversão", são realizadas na sessão as práticas "respiração na posição sentada", "espaço de respiração de 3 minutos adicional" e "cominar consciente", seguidas do inquiry. Um dos principais temas abordados é a aprender a ver os sentimentos negativos com menos aversão, ao entender que os sentimentos ruins e as mudanças sentidas são sintomas da depressão, e não um sinal de falha pessoal ou inadequação. O protocolo apresenta para a semana as 
práticas: "respiração na posição sentada", "espaço de respiração de 3 minutos", "espaço de respiração de 3 minutos adicional" e "cominar consciente".

Na quinta sessão do protocolo, de temática "Deixe as Coisas Serem Como Elas Já São", são realizadas em sessão as práticas "respiração na posição sentada: trabalhando com as dificuldades" e "espaço de respiração de 3 minutos responsivo", seguidas do inquiry. 0 pressuposto principal é que, ao mudar a postura diante da experiência de "não querer" para "estar aberto", há uma quebra nas reações automáticas. O protocolo propõe para a semana as práticas "respiração na posição sentada: trabalhando com as dificuldades", "espaço de respiração de 3 minutos regular", "espaço de respiração de 3 minutos responsivo" com instruções adicionais.

Na sexta sessão do protocolo, de tema "Veja os Pensamentos Como Pensamentos", tem como foco a ideia de que pensamentos não são fatos. Na sessão é realizado o exercício das "Três maneiras de praticar a percepção de praticar os pensamentos como eventos mentais". O protocolo propõe praticamente as mesmas atividades da semana anterior, complementadas com "Configuração de um Sistema de Advertência Precoce".

Na sétima sessão do protocolo, de temática "A Bondade em Ação", é realizada uma prática do "espaço de respiração de 3 minutos", seguida de inquiry. O protocolo trabalha com as mesmas três atividades da sexta semana, adicionando um Plano de Ação. Um dos tópicos da semana é a relação entre as ações e os sentimentos, sendo que é possível mudar o que é sentido através da mudança das ações. Como solicitado durante a sessão, o paciente constrói um plano de ação para os momentos em que perceber que está perdendo o controle. Primeiro, descreve os sinais que antecedem a desordem: pensamentos pessimistas, insegurança, irritação e depois inclui práticas de mindfulness e atividades prazerosas.

A oitava sessão do protocolo, de tema "E Agora?", trabalha a identificação dos benefícios percebidos durante o processo e como continuá-lo após o término dos encontros.

\section{Resultados}

A Tabela 1 mostra os dados sociodemográficos dos participantes.

A comparação entre os resultados das medidas antes e depois da intervenção e percentual de dedicação às práticas em sessão e em casa são apresentados na tabela 2. Este último item de comparação foi estabelecido considerando o número de estudos que demonstram que os benefícios de qualquer programa baseado em mindfulness são diretamente proporcionais à dedicação do participante às práticas no cotidiano, fora da sessão.
Tabela 1 - Dados sociodemográficos dos participantes, Foz do Iguaçu, PR, Brasil, 2018

\begin{tabular}{lccc}
\hline & Total & Completo & Desistência \\
\hline $\mathrm{N}$ & 12 & 11 & 1 \\
Idade média (anos) & 36,09 & 31 & 25 \\
Desvio Padrão (DP) & $(11,81)$ & $(12,10)$ & - \\
Sexo & & & \\
$\quad$ Feminino & 6 & 5 & 1 \\
$\quad$ Masculino & 6 & 6 & 0 \\
Escolaridade & & & \\
$\quad$ Ensino Superior incompleto & 8 & 7 & 1 \\
$\quad$ Ensino Superior completo & 3 & 3 & - \\
Fuma & 2 & 2 & - \\
Bebe & 6 & 5 & 1 \\
Faz uso de medicamento & 5 & 3 & 2 \\
Uso de medicamento psiquiátrico & 4 & 3 & 1 \\
Acompanhamento psiquiátrico & 2 & 2 & - \\
Realiza tratamento psicológico & 1 & 1 & - \\
Faz atividade física & 4 & 3 & 1 \\
\hline
\end{tabular}

Tabela 2 - Avaliações pré/pós dos participantes da pesquisa, Foz do Iguaçu, PR, Brasil, 2018

\begin{tabular}{lcccccc}
\hline & \multicolumn{2}{c}{ Pré-intervenção } & \multicolumn{2}{c}{ Pós-intervenção } & \multicolumn{3}{c}{ Anova* $^{*}$ Pré/Pós } \\
\cline { 2 - 7 } & $\mathbf{M}^{\dagger}$ & $\mathbf{D P}^{\ddagger}$ & $\mathbf{M}^{\dagger}$ & $\mathbf{D P}^{\ddagger}$ & $\mathbf{F}^{\S}$ & $\mathbf{p}^{\|}$ \\
\hline DASS-21 & & & & & & \\
Depressão & 86,75 & 0,92 & 62,67 & 14,64 & 29,72 & 0,0003 \\
Ansiedade & 83,25 & 8,82 & 68,58 & 9,68 & 18,71 & 0,0015 \\
Estresse & 82,17 & 7,81 & 36,17 & 14,90 & 199,15 & 6,27 \\
MAAS $^{* *}$ & 1,83 & 0,63 & 4,00 & 0,81 & 58,78 & 0,000017 \\
Práticas & & & 78,17 & 16,79 & & \\
\hline
\end{tabular}

*ANOVA (Fator duplo sem repetição); ${ }^{+} \mathrm{M}=$ Média aritmética; ${ }^{\ddagger} \mathrm{D}$ : Desvio Padrão; ${ }^{\mathrm{F}} \mathrm{F}$ = F Calculado; "Valor p; "DASS-21 = Depression, Ansiety and Stress Scale $; *$ MAAS $=$ Mindful Attention Awareness Scale

Os resultados demonstram que os 11 participantes apresentaram redução significativa dos sintomas de depressão, bem como dos níveis de ansiedade e estresse, condizente com a-os dados apresentados na literatura(6,14,25,27). Este dado pode ser observado nos dados da subescala depressão pré e pós intervenção $F(4,96)=29,72$ e $p=0,0003$, nos dados da subescala ansiedade pré e pós intervenção $F(4,96)=18,70$ e $p=0,0015$ e nos dados da subescala estresse pré e pós intervenção $F(4,96)=29,72$ e p = 0,0003 na Tabela 2 .

Os índices de maior remissão de sintomas de depressão, ansiedade e estresse está entre os participantes $1,2,3,4$ e 11 . Estes foram também os pacientes que mais realizaram as práticas fora da sessão. Estudiosos ${ }^{(14)}$ salientam que na adaptação do protocolo de 8 semanas original(2) provavelmente necessitar-se-á manter a ênfase na realização das tarefas e práticas meditativas de casa. Estudos apontam que as práticas em casa são mais importantes do que o próprio formato do programa, por exemplo, se realizado em 4 ou 8 semanas $^{(28)}$. 


\section{Discussão}

Em relação aos resultados da Escala MAAS, na avaliação pré-intervenção, todos os participantes apresentaram pontuação entre 1 e 3 que indica baixo nível de atenção no presente momento, indicando também possível frequentes distrações, envolvimento com preocupações com o futuro e ruminações acerca do passado. Já na avaliação pós intervenção, a maioria dos participantes elevou a atenção para o nível 4 ou 5 da escala. Houve um efeito significativo no estado de mindfulness entre a pré-intervenção e a pós, expressos nos dados de $\mathrm{F}(4,96)=58,78$ e $\mathrm{p}=0,000017$ na tabela 2. O grupo de pesquisadores da MBCT em formato individual(6,13,14-16-26,29-30) encontraram em seus estudos dados similares ao aumento da atenção ao momento presente. A partir do nível 4 da escala, o respondente apresenta boa atenção no momento, no qual a maioria de atividades que ele realiza recebem um bom grau de sua concentração e que refletem na vida afetiva, social e profissional. Já no nível 5 da escala, a atenção nas atividades simples e complexas é bem elevado e a relação com os eventos mentais como frequentes distrações, envolvimento com preocupações com o futuro e ruminações acerca do passado são muito menos frequentes, o que promove estado de bem-estar na maior parte do tempo. Vale ressaltar novamente que os participantes que mantiveram abaixo de 4 pontos na Escala MAAS (participantes 9 e 10) também foram os que menos se dedicaram às práticas fora da sessão.

Também importante dado relacionado à dedicação às práticas dos participantes é que aqueles que mais se dedicaram obtiveram melhores níveis de melhora dos sintomas de depressão, ansiedade e estresse, bem como de atenção ao momento presente, necessitando menor número de sessões para o progresso no programa. Isto reforça o já apontado que as práticas de casa representam, senão a maior responsável, uma das principais fontes dos benefícios do programa(28). São eles os participantes $1,2,3,4,5$ e 11 . O participante 10 também necessitou menor número de sessões, mas vale ressaltar que este participante tinha limitação de tempo para o programa, visto que estava com viagem marcada para o exterior, o que o fez se dedicar às sessões com maior afinco, mesmo que não às práticas de casa. Este participante trabalha viajando e este não foi um fator que pudesse influenciar positiva ou negativamente, visto que este tipo de viagem fazia parte do seu cotidiano.

Quanto à comorbidades, vale ressaltar que os participantes 1, 2, 3 e 7 preenchiam critérios do Transtorno de Personalidade Obsessivo-Compulsiva e foram submetidos também à avaliações específicas para este transtorno, apresentando melhora significativa dos sintomas. Por sua vez, os participantes 4, 5 e 10 preenchiam critérios do Transtorno de
Ansiedade Generalizada e foram submetidos também às avaliações específicas para este transtorno, apresentando os participantes 4 e 10 melhora significativa dos sintomas e o participante 5 a remissão completa dos sintomas. Enquanto, a participante 11 preenchia critérios de anorexia nervosa e foi submetida também à avaliações específicas para este transtorno, apresentando completa remissão dos sintomas. O grupo de pesquisadores da MBCT em formato individual(6,13-16,26,30) também mencionam comorbidades nas amostras pesquisadas, as quais merecem um novo aprofundamento em novas pesquisas.

Os resultados, tomados em conjunto, sugerem que a Terapia Cognitiva Baseada em Mindfulness pode ser utilizada no tratamento de pacientes com episódios recorrentes de depressão, mas também para ansiedade e estresse, corresponde ao apontado na literatura(2,6,13,27). A adaptação do protocolo de grupo para o contexto de atendimentos clínicos individuais se mostrou viável, tento em vista diferentes fatores. Em primeiro lugar devido à redução de sintomas, observado nos resultados das intervenções nos casos deste estudo, similar ao apresentado pela literatura para o clássico protocolo de grupo ${ }^{(2,5,9-10,13)}$. Em segundo lugar, devido ao aumento da adesão ao tratamento, visto que em contexto individual diminui a dispersão do contato com o instrutor e aumenta o vínculo. Os pacientes podem se sentir cuidados com maior atenção, sendo atendidos de maneira personalizada, conferindo com o proposto pela literatura(18-19). Em terceiro, devido à personalização da intervenção, considerando os aspectos particulares de cada paciente. Esta vantagem é apontada também na literatura(18-19).

Um estudo deste tipo apresenta algumas limitações. Em primeiro lugar, em termos de validade externa, uma vez que ele incluiu experimentos separados e com amostra de apenas onze casos, o que dificulta generalizações. Além disso, os participantes foram selecionados de maneira não aleatória, o que também enfraquece a validade externa. A validade externa de estudos com este delineamento pode ser reforçada com sua replicação várias vezes, fortalecendo possibilidades de generalizações.

\section{Conclusão}

Um crescente número de terapeutas vem utilizando a Terapia Cognitiva Baseada em Mindfulness adaptando o protocolo de grupos para o contexto clínico de indivíduos. Este estudo sugere que é possível manter o potencial de eficácia desta intervenção presente nas evidências científicas, mas exige adaptações. Além disso, ele reforça literatura de apoio à eficácia da Terapia Cognitiva Baseada em Mindfulness para depressão, ansiedade e estresse. 
Todos os participantes do estudo tiveram melhora significativa dos sintomas de depressão, bem como dos níveis de ansiedade e estresse, condizente com a os dados apresentados na literatura.

Um dado importante é que 6 dos 11 participantes que obtiveram maiores índices remissão dos sintomas de depressão, ansiedade e estresse foram também os pacientes que mais realizaram as práticas fora da sessão. A literatura aponta o caráter essencial das práticas de casa.

Estudos de MBCT aplicada ao contexto clínico individual são escassos na literatura. Foram encontrados apenas 04 estudos sobre a temática nas buscas para redação do presente estudo, contudo não há estudos de revisão que evidenciem a expressividade desses estudos na literatura científica. Desta forma, é premente a necessidade de maior número de estudos desta natureza.

Pesquisas futuras, especialmente as de natureza empírica, controlados e randomizados, consolidarão a validade externa dos resultados encontrados por estudos com a natureza como o deste. O aumento deste tipo de estudos possibilitará análises estatísticas que constroem a evidência da efetividade da intervenção, possibilitando que sejam aplicadas e estimuladas quanto à sua utilização no contexto clínico individual.

\section{Agradecimentos}

Aos pacientes que se disponibilizaram suas histórias e aceitaram participar da pesquisa.

\section{Referências}

1. Barber RM, Fullman N, Sorensen RJD, Bollyky T, McKee $M$, Nolte $E$, et al. Healthcare Access and Quality Index based on mortality from causes amenable to personal health care in 195 countries and territories; 1990-2015: a novel analysis from the Global Burden of Disease Study 2015. Lancet. [Internet]. 2017; 390(10091):31-66. https://doi.org/10.1016/S0140-6736(17)30818-8

2. Segal ZV, Williams JMG, Teasdale JD. Mindfulnessbased cognitive therapy for depression: A new approach to preventing relapse. 2nd ed. New York (NY): Guilford Press; 2012.

3. Beck AT, Rush AJ, Shaw BF, Emery G. Cognitive therapy of depression. New York; NY: Guilford Press; 1979.

4. Kabat-Zinn J. Full catastrophe living: Using the wisdom of your mind to face stress; pain; and illness. New York(NY): Bantam Dell; 1990.

5. Kuyken W, Watkins E, Holden E, White K, Taylor RS, Byford $S$, et al. How does mindfulness-based cognitive therapy work? Behav Res Therapy. [Internet]. 2010; 48(11):1105-12. Available from: https://www.ncbi.nlm. nih.gov/pubmed/20810101.
6. Williams JM, Kuyken W. Mindfulness-based cognitive therapy: a promising new approach to preventing depressive relapse. The British Journal of psychiatry: The Journal of Mental Science. [Internet]. 2012; 200(5):359-60. Available from: https://www.ncbi.nlm. nih.gov/pubmed/22550328.

7. Kuyken W, Warren F, Taylor R, Whalley B, Crane C, Bondolfi G. Efficacy of Mindfulness-Based Cognitive Therapy in Prevention of Depressive Relapse. JAMA Psychiatry. [Internet]. 2016; 73(6):565-74. Available from: https://www.ncbi.nlm.nih.gov/pubmed/27119968. 8. National Institute of Health and Clinical Excellence. http://www.nice.org.uk/.jsp [cited Mar 12, 2009].

9. Piet J, Wurtzen H, Zachariae R. The effect of mindfulness-based therapy on symptoms of anxiety and depression in adult cancer patients and survivors: a systematic review and meta-analysis. J Consult Clin Psychol. [Internet]. 2012; 80(6):1007-20. Available from: https://www.ncbi.nlm.nih.gov/pubmed/22563637. 10. Fjorback LO, Arendt M, Ornbol E, Fink P, Walach $H$. Mindfulness-based stress reduction and mindfulnessbased cognitive therapy-a systematic review of randomized controlled trials. Acta Psychiatr Scand. [Internet]. 2011; 124(2):102-19. Available from: https://www.ncbi.nlm.nih.gov/pubmed/21534932.

11. Hofmann SG, Sawyer AT, WittAA, Oh D. The effect of mindfulness-based therapy on anxiety and depression: a meta-analytic review. J Consult Clin Psychol. [Internet]. 2010; 78(2):169-83. Available from: https://www.ncbi. nlm.nih.gov/pubmed/20350028.

12. Beltman MW, Voshaar RC, Speckens AE. Cognitivebehavioural therapy for depression in people with a somatic disease: meta-analysis of randomised controlled trials. Br J Psychiatr. [Internet]. 2010; 197(1):119. Available from: https://www.ncbi.nlm.nih.gov/ pubmed/20592427.

13. Chiesa A, Serretti A. Mindfulness-based stress reduction for stress management in healthy people: a review and meta-analysis. J Alternative Complem Medicine. [Internet]. 2009; 15(5):593-600. Available from: https://www.ncbi.nlm.nih.gov/pubmed/19432513. 14. Schroevers MJ, Tovote KA, Keers JC, Links TP, Sanderman R, Fleer J. Individual mindfulness-based cognitive therapy for people with diabetes: a pilot randomized controlled trial. Mindfulness. [Internet]. 2015; 6(1):99-110. Available from: https://link. springer.com/article/10.1007/s12671-013-0235-5.

15. Tovote KA, Fleer J, Snippe E, Peeters ACTM, Emmelkamp PMG, Sanderman $R$, et al. Individual mindfulness-based cognitive therapy and cognitive behavior therapy for treating depressive symptoms in patients with diabetes: results of a randomized controlled trial. Diabetes Care. [Internet]. 2014; 37(9):2427-34. Available from: https://www.ncbi.nlm. nih.gov/pubmed/24898301

16. Tovote KA, Schroevers MJ, Snippe E, Sanderman R, Links TP, Emmelkamp PMG, et al. Long-Term Effects 
of Individual Mindfulness-Based Cognitive Therapy and Cognitive Behavior Therapy for Depressive Symptoms in Patients with Diabetes: A Randomized Trial. Psychother Psychosom. [Internet]. 2015; 84:186-7 Available from: https://www.ncbi.nlm.nih.gov/pubmed/25832365.

17. Dwight-Johnson M, Sherbourne C, Liao D, Wells K. Treatment preferences among depressed primary care patients. J Gen Internal Med. [Internet]. 2000; 15(8):527-34. Available from: https://www.ncbi.nIm. nih.gov/pmc/articles/PMC1495573/.

18. Lau MA, Colley L, Willett BR, Lynd LD. Employee's preferences for access to mindfulness-based cognitive therapy to reduce the risk of depressive relapse-a discrete choice experiment. Mindfulness. [Internet]. 2012; 3:318-26. Available from: https://link.springer. com/article/10.1007/s12671-012-0108-3.

19. Wahbeh H, Lane JB Goodrich E, Miller M, Oken BS. One-on-one mindfulness meditation trainings in a research setting. Mindfulness. [Internet]. 2014; 5(1):88-99. Available from: https://www.ncbi.nlm.nih. gov/pubmed/24554970.

20. Yin RK. Estudo de caso: planejamento e métodos. 2 ed. Porto Alegre(RS): Bookman; 2001.

21. Fachin O. Fundamentos de metodologia. São Paulo(SP): Saraiva; 2001.

22. Miles MB, Huberman AM. Qualitative Data Analysis: An Expanded Sourcebook. 2nd.ed. Thousand Oaks/ London: Sage; 1994.

23. First MB, Williams JBW, Spitzer RL. Entrevista clínica estruturada para os transtornos do DSM-5: SCID. SCV versão clínica. Porto Alegre. Artemed; 2017.

24. Vignola RCB, Tucci AM. Adaptation and validation of the Depression, Anxiety and Stress Scale (DASS) to Brazilian portuguese. J Affect Disord. 2014;155:1049. Available from: http://repositorio.unifesp.br/ handle/11600/37373.

25. Barros VV, Kozasa EH, Souza IW, Ronzani TM. Validity evidence of the brazilian version of the Mindful Attention Awareness Scale (MAAS). Psicol Reflexão Crít. [Internet]. 2015; 28(1):87-95. Available from: http://www.scielo.br/scielo.php?pid=S010411692006000600006\&script=sci_arttext\&tIng=pt.

26. Teasdale JD, Segal, ZV, Williams JM, Ridgeway VA, Soulsby JM, Lau MA. Prevention of relapse/recurrence in major depression by mindfulness-based cognitive therapy. J Consult Clin Psychol. [Internet]. 2000; 68:615-23. Available from: https://www.ncbi.nlm.nih. gov/pubmed/10965637.
27. Geschwind N, Peeters F, Huibers M, Van OJ, Wichers, M. Efficacy of mindfulness-based cognitive therapy in relation to prior history of depression: randomised controlled trial. The $\mathrm{Br}$ J Psychiatr. [Internet]. 2012; 201(4):320-5. Available from: https://www.ncbi.nlm. nih.gov/pubmed/22878133.

28. Demarzo M, Montero-Marin J, Puebla-Guedea M, Navarro-Gil M, Herrera-Mercadal P, Moreno-González S, et al. Efficacy of 8- and 4-Session Mindfulness-Based Interventions in a Non-clinical Population: A Controlled Study. Frontiers Psychol. [Internet]. 2017; 8:13-43. http://doi.org/10.3389/fpsyg.2017.01343 Available from: https://www.ncbi.nlm.nih.gov/pubmed/28848465. 29. Tovote KA, Schroevers MJ, Snippe E, Sanderman R, Links TP, Emmelkamp PMG, et al. What works best for whom? Cognitive Behavior Therapy and MindfulnessBased Cognitive Therapy for depressive symptoms in patients with diabetes. PLoS ONE. [Internet]. 2017; 12(6): e0179941. Available from: https://www.ncbi.nlm. nih.gov/pubmed/28662208.

30. Segal ZV, Williams JMG, Teasdale JD. The Mindful Way Workbook - An 8-Week Program to Free Yourself from Depression and Emotional Distress. New York (NY): The Guilford Press; 2014.

\section{Contribuição dos autores}

Concepção e planejamento do estudo: Nazaré de Oliveira Almeida, Marcelo Demarzo e Carmem Beatriz Neufeld. Obtenção dos dados: Nazaré de Oliveira Almeida. Análise e interpretação dos dados: Nazaré de Oliveira Almeida, Marcelo Demarzo e Carmem Beatriz Neufeld. Análise estatística: Nazaré de Oliveira Almeida, Marcelo Demarzo e Carmem Beatriz Neufeld. Obtenção de financiamento: Nazaré de Oliveira Almeida e Carmem Beatriz Neufeld. Redação do manuscrito: Nazaré de Oliveira Almeida, Marcelo Demarzo e Carmem Beatriz Neufeld. Revisão crítica do manuscrito: Nazaré de Oliveira Almeida, Marcelo Demarzo e Carmem Beatriz Neufeld.

Todos os autores aprovaram a versão final do texto.

Conflito de interesse: os autores declararam que não há conflito de interesse.
Copyright $\odot 2020$ SMAD, Rev. Eletrônica Saúde Mental Álcool Drog. Este é um artigo de acesso aberto distribuído sob os termos da Licença Creative Commons CC BY-NC.

Esta licença permite que outros remixem, adaptem e criem a partir do seu trabalho para fins não comerciais, e embora os novos trabalhos tenham de Ihe atribuir o devido crédito e não possam ser usados para fins comerciais, os usuários não têm de licenciar esses trabalhos derivados sob os mesmos termos. 